\title{
On the deleted product criterion for embeddability of manifolds in $\mathbb{R}^{m}$
}

\author{
A. Skopenkov*
}

\begin{abstract}
For a space $N$ let $\tilde{N}=\{(x, y) \in N \times N \mid x \neq y\}$. Let $\mathbb{Z}_{2}$ act on $\tilde{N}$ and on $S^{m-1}$ by exchanging factors and antipodes, respectively. For an embedding $f: N \rightarrow \mathbb{R}^{m}$ define the map $\tilde{f}: \tilde{N} \rightarrow S^{m-1}$ by $\tilde{f}(x, y)=\frac{f x-f y}{|f x-f y|}$.

Theorem. Let $d=3 n-2 m+2$ and $N$ be a closed $P L$ n-manifold.

a) If $d \in\{0,1,2\}, N$ is $d$-connected and there exists an equivariant map $F: \tilde{N} \rightarrow S^{m-1}$, then $N$ is $P L$-embeddable in $\mathbb{R}^{m}$.

b) If $d \in\{-1,0,1\}, m-n \geq 3, N$ is $(d+1)$-connected and $f, g: N \rightarrow \mathbb{R}^{m}$ are $P L$ embeddings such that $\tilde{f}, \tilde{g}$ are equivariantly homotopic, then $f, g$ are $P L$-isotopic.

Corollary. a) Every closed 6-manifold $N$ such that $H_{1}(N)=0$ PL embeds in $\mathbb{R}^{10}$; b) Every closed PL 2-connected 7-manifold PL embeds in $\mathbb{R}^{11}$; $(l>0)$.

c) There are exactly four $P L$ embeddings $S^{2 l+1} \times S^{2 l+1} \subset \mathbb{R}^{6 l+4}$ up to $P L$ isotopy
\end{abstract}

Mathematics Subject Classification (1991). Primary: 57Q35, 57Q37; secondary: 54C25, 55S15, 57Q30, 57Q60, 57Q65.

Keywords. Embedding, deleted product, engulfing, singular set, normal Whitney classes, quasiembedding, metastable case, highly-connected manifolds, isotopy, concordance.

\section{Introduction}

To find necessary and sufficient conditions for a manifold $N$ to be embeddable in $\mathbb{R}^{m}$ for a given $m$ is a classical problem in topology (see [Wu 65, RS 96]). All manifolds and their embeddings are supposed to be PL, unless the contrary is stated. Let $\tilde{N}=\{(x, y) \in N \times N \mid x \neq y\}$ be the deleted product of $N$ (cf. [Va 92]). Let $\mathbb{Z}_{2}$ act on $S^{m-1}$ by exchanging factors and antipodes respectively. If $f: N \rightarrow \mathbb{R}^{m}$ is an embedding then there exists an equivariant map $\tilde{f}: \tilde{N} \rightarrow S^{m-1}$, defined by $\tilde{f}(x, y)=\frac{f(x)-f(y)}{\|f(x)-f(y)\|}$. If $f_{t}: K \rightarrow \mathbb{R}^{m}$ is an isotopy between embeddings

\footnotetext{
* Supported in part by the Russian Fundamental Research Foundation Grant No 96-0101166A, and the International Science Foundation Grant No a97-586.
} 
$f_{0}, f_{1}: N \rightarrow \mathbb{R}^{m}$, then there exists an equivariant homotopy $\tilde{f}_{t}$ between $\tilde{f}_{0}$ and $\tilde{f}_{1}$. However trivial these necessary conditions may seem, they are very useful [Hae $63, \mathrm{Wu} 65, \mathrm{We} 67]$. Thus it is very interesting to find out, for which cases they are also sufficient for embeddability and isotopy.

Theorem 1.0. ([Hae 63, We 67] see also [Har 69]) Suppose that $N$ is either a Diff $n$-manifold or an $n$-polyhedron and $m \geq \frac{3(n+1)}{2}$. Then

a) $N$ is $P L(D i f f)-e m b e d d a b l e$ in $\mathbb{R}^{m}$ if and only if there exists an equivariant map $F: \tilde{N} \rightarrow S^{m-1}$.

b) (realization version) Moreover, for each equivariant map $F: \tilde{N} \rightarrow S^{m-1}$ there exists an embedding $f: N \rightarrow \mathbb{R}^{m}$ such that $\tilde{f}$ is equivariantly homotopic to F.

c) (boundary version) Suppose that $A$ is a subpolyhedron of $N, B^{m}$ is a $P L$ $m$-ball, $g: N \rightarrow B^{m}$ is a PL-map such that $\left.g\right|_{A}$ is an embedding into $\partial B^{m}$ and $g(N-A) \subset \stackrel{\circ}{B}^{m}$. There exists an embedding $f: N \rightarrow B^{m}$ such that $\left.f\right|_{A}=\left.g\right|_{A}$ and $f(N-A) \subset \stackrel{\circ}{B}^{m}$ if and only if the equivariant map $\tilde{g}: A^{*} \rightarrow S^{m-1}$ homotopically extends to an equivariant map $F: \tilde{N} \rightarrow S^{m-1}$.

d) (relative version) Suppose that $A$ is a subpolyhedron of $N$ and $g: A \rightarrow \mathbb{R}^{m}$ is a PL-embedding. There exists an embedding $f: N \rightarrow \mathbb{R}^{m}$ such that $\left.f\right|_{A}=g$ if and only if the equivariant map $\tilde{g}: \tilde{A} \rightarrow S^{m-1}$ homotopically extends to an equivariant map $F: \tilde{N} \rightarrow S^{m-1}$.

e) (isotopy version) Suppose that $m>\frac{3(n+1)}{2}$. Then $P L$ (Diff)-embeddings $f_{0}$, $f_{1}: N \rightarrow \mathbb{R}^{m}$ are PL(Diff)-isotopic if and only if the equivariant maps $\tilde{f}_{0}, \tilde{f}_{1}$ are equivariantly homotopic.

For a subpolyhedron $A \subset N$ we denote $A^{*}=\tilde{A} \cup[A \times(N-A)] \cup[(N-A) \times A]$. Note that the case $(m, n)=(3,1)$ of theorem 1.0.b is not proved in [We 67], but can be proved in the same ideas [Sk]. Theorem 1.0 can be summarized as follows: the map $f \rightarrow \tilde{f}$ from the set of embeddings of $N \rightarrow \mathbb{R}^{m}$ up to isotopy to the set of equivariant maps $\tilde{N} \rightarrow S^{m-1}$ up to equivariant homotopy is a 1-1 correspondence for $m>\frac{3(n+1)}{2}$ and a surjection for $m=\frac{3(n+1)}{2}$. It turns out that the dimension restriction $m \geq \frac{3(n+1)}{2}\left(m>\frac{3(n+1)}{2}\right)$ is sharp in theorem 1.0.a (1.0.e) for polyhedra [SSS 97, MS 67, Hus 88, SS 92, FKT 94] (diffeotopy of connected Diff-manifolds [Ha 62] and PL isotopy of non-connected PL-manifolds [Ma 90, Proposition 8.3]). But these dimension restrictions can be weakened for connected PL manifolds. Many corollaries of the Haeflinger-Weber theorem are thus also strengthened.

Theorem 1.1. a) Suppose that $N$ is a closed $P L$ n-manifold and either $m=$ $\frac{3 n}{2}+1$, or $N$ is simply connected and $m=\frac{3 n+1}{2}$, or $N$ is 2-connected and $m=\frac{3 n}{2}$. Then $N$ is $P L$ embeddable in $\mathbb{R}^{m}$ if and only if there exists an equivariant map $F: \tilde{N} \rightarrow S^{m-1}$. 
b) (boundary version) Suppose that $N$ is a PL n-manifold (possibly, nonclosed) and either $N$ is connected and $m=\frac{3 n}{2}+1 \geq 7$, or $N$ is simply connected and $m=\frac{3 n+1}{2} \geq 8$, or $N$ is 2-connected and $m=\frac{3 n}{2} \geq 9$. Suppose further that $A$ is a subpolyhedron of $N$, containing $\partial N, B^{m}$ is a PL m-ball, $g: N \rightarrow B^{m}$ is a PL-map such that $\left.g\right|_{A}$ is an embedding into $\partial B^{m}$ and $g(N-A) \subset \stackrel{\circ}{B^{m}}$. There exists an embedding $f: N \rightarrow B^{m}$ such that $\left.f\right|_{A}=\left.g\right|_{A}$ and $f(N-A) \subset \stackrel{\circ}{B^{m}}$ if and only if the equivariant map $\tilde{g}: A^{*} \rightarrow S^{m-1}$ extends to an equivariant map $F: \tilde{N} \rightarrow S^{m-1}$.

c) (isotopy version) Suppose that $N$ is a closed $P L$ n-manifold and either $N$ is connected and $m=\frac{3 n+3}{2} \geq 6$, or $N$ is simply connected and $m=\frac{3 n}{2}+1 \geq 7$, or $N$ is 2-connected and $m>\frac{3 n+1}{2} \geq 8$. Then PL-embeddings $f_{0}, f_{1}: N \rightarrow \mathbb{R}^{m}$ are $P L$-isotopic if and only if equivariant maps $\tilde{f}_{0}, \tilde{f}_{1}$ are equivariantly homotopic.

We prove theorem 1.1.a and its corollaries for $m \geq n+3$. For $m<n+3$ they are known. Boundary version 1.1.b is necessary to prove the isotopy version 1.1.c. From our proof it follows that theorem 1.1.a (1.1.c) and corollary 1.2.abc (1.2.d) are true even if there exists an equivariant map to $S^{m-1}$ only from the $\left(\left[\frac{4 m}{3}\right]-2\right.$ )-skeleton (equivariant maps are homotopic only on $\left[\frac{4 m-5}{3}\right]$-skeleton) of $\tilde{N}$, and $N$ has singularities of dimension at most $m-n-2$.

Corollary 1.2. Under assumptions of theorem 1.1.a,

a) embeddability of $N$ into $\mathbb{R}^{m}$ does not depend on PL structure on $N$;

b) if $N$ is Top-embeddable in $\mathbb{R}^{m}$, then $N$ is $P L$-embeddable in $\mathbb{R}^{m}$;

c) if $N$ is quasi-embeddable in $\mathbb{R}^{m}$, then $N$ is embeddable in $\mathbb{R}^{m}$;

d) under assumptions of theorem 1.1.c, if two PL-embeddings $f, g: N \rightarrow \mathbb{R}^{m}$ are Top-isotopic, then they are PL-isotopic.

A polyhedron $N$ is called quasi-embeddable in $\mathbb{R}^{m}$ if for each of its triangulations $T$ there exists a map $f: N \rightarrow \mathbb{R}^{m}$ (which is called an almost-embedding [FKT 94]) such that $f \sigma \cap f \tau=\emptyset$ for each $\sigma \times \tau \in \widetilde{T}, \sigma \cap \tau=\emptyset$. This definition is non-standard, but equivalent to the standard one: for each $\varepsilon>0$ there is a map $f: N \rightarrow \mathbb{R}^{m}$ whose preimages are of diameter less than $\varepsilon$.

Corollary 1.3. a) (cf. [Bo 71, theorem 7.4, erroneously named in the introduction as theorem 7.1], for $k=2$ cf. [Hi 65, BH 70, Fu 94, main theorem ii]) $A$ homologically $(k-2)$-connected closed PL $2 k$-manifold $N$ embeds in $\mathbb{R}^{3 k+1}$ if and only if $\bar{W}_{k+1}(N)=0$.

b) (for $k=1$ [Hi 61, Ro 65, Wa 65], for $k=2$ cf. [Hi 61]) $A(k-1)$-connected closed $P L(2 k+1)$-manifold $N$ embeds in $\mathbb{R}^{3 k+2}$ if and only if $\bar{W}_{k+1}(N)=0$.

c) (cf. [Mi 65], for $k=2[\mathrm{CS} 79, \mathrm{Ru} 82]) A(k-1)$-connected closed $P L$ $2 k$-manifold $N$ embeds in $\mathbb{R}^{3 k}$ if and only if $\bar{W}_{k}(N)=0$.

Here $\bar{W}_{i}(N)$ denotes the $i$-th normal Stiefel-Whitney class of $N$ with coefficients $\mathbb{Z}$ (if $i>1$ is odd and $N$ is orientable), $\mathbb{Z}$ twisted (if $i>1$ is odd and $N$ 
is non-orientable) or $\mathbb{Z}_{2}$ (if $i=1$ or is even). Corollary 1.3 follows from theorem 1.1.a and [We 67, theorem on p.4], cited from [Hae 62]. Note that for $n \geq 5$, $m<\frac{3 n}{2}$, a $(2 n-m-1)$-connected closed $n$-manifold is a PL sphere, and therefore embeds into $\mathbb{R}^{n+1}$. From [We 67, theorem 4, Hae 63] and [Ma 60, corollary 2] it follows that if $n \geq 8$ is even and $n \neq 2^{k}\left(2^{h}+1\right)$ for each integers $k, h \geq 2$, then every PL or Diff $n$-manifold $N$ such that $H_{1}(N)=0$, PL of Diff embeds in $\mathbb{R}^{2 n-2}$. Corollary 1.3.a for $k=2,3$, corollary 1.3.b for $k=3$ and [Ma 62, theorem 1], [Ma 60 , corollary 2, theorem 1c] imply the following (from the proof [Ma 60, p. 100] follows that the restriction [Ma 60, thorem 1c] should be stated as 'the number of $h_{i}$ 's which are equal to $h_{q}+1$ is even').

Corollary 1.4. a) [Hi 65] Every orientable 4-manifold PL embeds in $\mathbb{R}^{7}$;

b) (cf. [Hi 61]) Every closed 6-manifold $N$ with $H_{1}(N)=0$ PL embeds in $\mathbb{R}^{10}$.

c) Every closed 2-connected PL 7-manifold PL embeds in $\mathbb{R}^{11}$.

In a) and b) it is not necessary to specify, which (PL or Diff) manifolds are under consideration, because every PL manifold of dimension at most 6 is smoothable. Note that every 2-connected closed 6-manifold is a disjoint sum of $S^{3} \times S^{3}[\mathrm{Sm}$ 62 , theorem $\mathrm{B}]$ and therefore is PL or Diff embeddable into $\mathbb{R}^{7}$. From the results of [Zh 75, Zh 80, Zh 89] it follows that there exists a 1-connected 6-manifold $N$ with $\bar{W}_{3}(N) \neq 0$, hence $N$ is non-embeddable in $\mathbb{R}^{9}$.

Corollary 1.5. (cf. [Hu 69, Ch.11.4]) a) For each $k>1$ and a connected orientable homologicaly $(k-2)$-connected closed $P L(2 k-1)$-manifold $N$, the set of $P L$ embeddings $N \rightarrow \mathbb{R}^{3 k}$ up to $P L$ isotopy is in 1-1 correspondence with $H_{k-1}\left(N, \mathbb{Z}_{(k)}\right)$.

b) For each $k>2$ and a $(k-1)$-connected closed $P L$ 2k-manifold $N$ there is a 1-1 correspondence between the set of its embeddings $N \rightarrow \mathbb{R}^{3 k+1}$ and $H_{k}\left(N, \mathbb{Z}_{(k)}\right)$.

c) For each non-orientable connected closed PL 3-manifold $N$, the set of $P L$ embeddings $N \rightarrow \mathbb{R}^{6}$ up to $P L$ isotopy is in $1-1$ correspondence with $H_{1}\left(N, \mathbb{Z}_{2}\right)$.

Here $\mathbb{Z}_{(k)}$ is $\mathbb{Z}_{2}$ for $k$ odd and $\mathbb{Z}$ for $k$ even. Note that the injectivity was not proved in [Hu 69, Ch.11.4.1,2]. For $k$ odd and for c) corollary 1.5 imply that the set of embeddings is finite. Corollary 1.5 follows from theorems 1.0.e, 1.1.c, $[\mathrm{Hu}$ 69, theorem 11.9 and 'onto' part of theorem 11.4.2] and [We 67, theorem on p. 5], cited from [Hae 62]. Note that for $n+3 \leq m<\frac{3 n}{2}+1$ we have $n \geq 5$, hence a $(2 n-m)$-connected closed $n$-manifold is a PL sphere and therefore has a unique PL embedding into $\mathbb{R}^{m}$. Corollaries 1.5.ab imply

Corollary 1.6. a) [Hu 63, theorem 1]) For each $k>1$ the set of $P L$ embeddings $S^{k} \times S^{k-1} \rightarrow \mathbb{R}^{3 k}$ up to PL isotopy is in 1-1 correspondence with $\mathbb{Z}_{(k)}$.

b) (cf. [Hu 63, corollary to theorem 1], [Hu 69, Ch.11.4]) For each $k>2$ there is a 1-1 correspondence between the set of $P L$ embeddings $S^{k} \times S^{k} \rightarrow \mathbb{R}^{3 k+1}$ (up to $P L$ isotopy) and $\mathbb{Z}_{(k)}+\mathbb{Z}_{(k)}$. 
Proof of Theorem 1.1.a is based on the extension of Weber's theorem, on the Penrose-Whitehead-Zeeman-Irwin theorem and on engulfings. Our proof does not yield relative, realization and approximation [We 67, theorem 3] versions of theorem 1.1 (approximation theorem was proved in [Ch 69, Mi 70, Br 72] even for embeddings of polyhedra in codimension at least 3 using different technique). Isotopy version 1.1.c follows from the boundary version 1.1.b and the "concordance implies isotopy' theorem [ $\mathrm{Li} 65, \mathrm{Hu} 70]$. Note that the relative version 1.0.c [We 67 , theorem 7a] is not sufficient to prove the isotopy version 1.0.e [We 67, theorem 67 , theorem $7 \mathrm{a}$ ] is not sufficient to prove the isotopy version 1.0.e [We 67, theorem $\left.1^{\prime}\right]$. For in [We 67, §7] we obtain an embedding $\varphi: K \times I \rightarrow \mathbb{R}^{m+1}$ but not $\varphi: K \times I \rightarrow \mathbb{R}^{m} \times I$. This is only a minor inaccuracy and we correct it in $\S 3$.

\section{Proof of theorem 1.1.a}

2.1. A reduction of theorem 1.1.a. Necessity in theorem 1.1.a was explained in the beginning of section 1, so we are to prove sufficiency. For PL-topology we follow the notation of [RS 72]. Since every 2-manifold embeds in $\mathbb{R}^{4}$, theorem 1.1.a is true for $m=\frac{3 n}{2}+1=4$. Since every 3 -manifold embeds in $\mathbb{R}^{5}$ [Hi 61, Ro 65, Wa 65], theorem 1.1.a is true for $m=\frac{3 n+1}{2}=5$. To see that theorem 1.1.a is true for $m=\frac{3 n}{2}=6$ recall that every 2 -connected closed 4-manifold is a homotopy sphere. By [Ker 69, corollary on p. 71] it is boundary of a conractible PL 5-manifold. By the Van Kampen theorem and from the Mayer-Vietoris sequence, its double is simply connected homology sphere. Hence it is a homotopy sphere [FF 89, 114 , exercise 6] and therefore a PL sphere [Ze 62]. Then this double minus a point is $\mathbb{R}^{5}$ in which $N$ is embedded. Therefore we may assume that $m \geq n+3$. The proof of theorem 1.1.a is based on the following results (which may be useful in attacking of conjecture 4.3.a).

Theorem 2.1.1. Let $N$ be an n-polyhedron with triangulation T. If $m-n \geq 3$ and there exists an equivariant map $F: \tilde{N} \rightarrow S^{m-1}$, then there exists a general position PL-map $f: N \rightarrow \mathbb{R}^{m}$ such that

a) $\left.f\right|_{\sigma}$ is an embedding for each $\sigma \in T$;

b) $f \sigma \cap f \tau=f(\sigma \cap \tau)$ whenever $\operatorname{dim} \tau \leq \operatorname{dim} \sigma$ and $2 \operatorname{dim} \sigma+\operatorname{dim} \tau \leq 2 m-3$.

The proof is analogous to [We $67, \S 5$, Sk $97, \S 2]$ : the inequality $2 m \geq 3(n+1)$ can be relaxed to $\operatorname{dim} \tau \leq \operatorname{dim} \sigma$ and $2 \operatorname{dim} \sigma+\operatorname{dim} \tau \leq 2 m-3$ keeping the proof unchanged (cf. [ST 91]).

Theorem 2.1.2. [Ze 62] Let $S$ be a k-polyhedron in the interior of an n-manifold $N$. If the inclusion $S \subset N$ is null-homotopic, $n-k \geq 3$ and $N$ is $(2 k-n+2)$ connected, then $S$ is contained in some PL n-ball in the interior of $N$. 
Theorem 2.1.3. Suppose that $N$ is a closed $(3 n-2 m+2)$-connected $P L n$ manifold, $m-n \geq 3$ and there is a map $f: N \rightarrow \mathbb{R}^{m}$ such that $S(f)=C l\{x \in N$ : $\left.\left|f^{-1} f x\right|>1\right\}$ is contained in some PL n-ball in $N$. Then $N$ is embeddable in $\mathbb{R}^{m}$.

Theorem 2.1.3. was actually proved in [PWZ 61, Ir 65, RS 72, theorem 7.12].

Let $d=3 n-2 m+2$. Take a small triangulation $T$ of $N$ and a map $f$ : $N \rightarrow \mathbb{R}^{m}$ given by theorem 2.2.1. By general position $\operatorname{dim} S(f) \leq 2 n-m$. Since $n-(2 n-m) \geq 3$ and $N$ is $2(2 n-m)-n+2=d$-connected, then by theorems 2.1.1 and 2.1.3 it sufficies to prove that the inclusion $S(f) \subset N$ is null-homotopic. Since $N$ is $d$-connected (for $d=0$ we may assume that $N$ is connected), it sufficies to prove that the inclusion $S(f) \subset N$ is homotopic to a map into some $d$-dimensional subpolyhedron of $N$.

2.2. Idea of the proof and proof of the case $d=0$. Take a map $f: N \rightarrow \mathbb{R}^{m}$ given by 2.2.1. If we assume additionally that $f$ is a PL immersion (i.e. local embedding), then by theorem 2.2.1., $S(f)$ does not intersect the $(2 m-2 n-3)$ skeleton of $T$. Hence it retracts to the $n-1-(2 m-2 n-3)=d$-skeleton of a triangulation, dual to $T$. But in general, $S(f)$ does intersect the $(2 m-2 n-3)$ skeleton of $T$. However, this approach works to prove the main theorem of [Sk].

Let us fix some notations. By small Greek letters we will denote simplices of $T$. Their dimension will be sometimes indicated as an upper index. For $\alpha, \beta \in T$ we denote by $\alpha \beta$ the ordered pair $(\alpha, \beta)$ when $\alpha \cap \beta=\emptyset$ and the non-ordered pair $\{\alpha, \beta\}$ when $\alpha \cap \beta \neq \emptyset, \alpha \not \subset \beta, \alpha \not \supset \beta$. For $\alpha \not \subset \beta, \alpha \not \supset \beta$ let

$$
\left(A_{\alpha \beta}, S_{\alpha \beta}\right)= \begin{cases}\left(\alpha, \alpha \cap f^{-1} f \beta\right), & \alpha \cap \beta=\emptyset \\ \left(\alpha \cup \beta, f^{-1} \mathrm{Cl}[(f \alpha \cap f \beta)-f(\alpha \cap \beta)]\right), & \alpha \cap \beta \neq \emptyset\end{cases}
$$

Since $\left.f\right|_{\sigma}$ is an embedding for each $\sigma$ and since each simplex of $T$ is contained in some $n$-dimensional one, then $S(f)=\bigcup\left\{S_{\alpha^{n} \beta^{n}} \mid \alpha^{n} \neq \beta^{n}\right\}$. Since $T$ is a triangulation then each $A_{\alpha \beta}$ is contractible.

Now suppose that $d=0$. By 2.1.1.b $S_{\alpha \beta} \neq \emptyset$ is possible only if $\operatorname{dim} \alpha=$ $\operatorname{dim} \beta=n$. Since that and by general position, $S_{\alpha \beta} \cap S_{\gamma \delta}=\emptyset$ when $\alpha \beta \neq \gamma \delta$. Therefore contractibility of $A_{\alpha \beta}$ implies that the inclusion $S(f) \subset N$ is homotopic to a map onto a finite subset of $N$.

The cases $d=1,2$ follow from 2. $(d+2)$ and 2.5 .

2.3. Decomposition of $S(f)$ for $d=1$. Let $N$ be a closed $P L$ n-manifold and $m=\frac{3 n+1}{2} \geq n+3$. Then there is a triangulation $T$ of $N$ such that for every map $f: N \rightarrow \mathbb{R}^{m}$, satisfying to (2.1.1.a,b) there are subpolyhedra $S_{1}, \ldots, S_{s} \subset N$ for which $S(f)=S_{1} \cup \cdots \cup S_{s}$ and

a) $S_{i} \cap S_{j} \cap S_{k}=\emptyset$ for distinct $i, j, k=1, \ldots, s$;

b) For each $i=1, \ldots, s$ there is a contractible polyhedron $A_{i} \subset N$, containing $S_{i}$. If $S_{i} \cap S_{j} \neq \emptyset$, then there is a contractible polyhedron $A_{i j} \subset N$, containing $A_{i} \cup A_{j}$. 
Proof. Take a triangulation $T$ of $N$ such that for each $x \in N$, the star $\operatorname{st}^{2} x=\operatorname{st}$ st $x$ is contractible. Enumerate by integers $1, \ldots, s$ the union of the set of ordered pairs of disjoint $n$-simplices of $T$ with the set of non-ordered pairs $\{\alpha, \beta\}$ of intersecting $n$-simplices of $T$ such that $\alpha \not \supset \beta$. Let $S_{1}, \ldots, S_{s}$ be as defined in 2.2. Then $S(f)=S_{1} \cup \cdots \cup S_{s}$.

By 2.1.1.b $S_{\alpha \beta} \neq \emptyset$ is possible only when either $\operatorname{dim} \alpha=\operatorname{dim} \beta=n$ or $\{\operatorname{dim} \alpha, \operatorname{dim} \beta\}=\{n, n-1\}$. By general position, $f$ has no triple points. Therefore each non-empty intersection of 3 of $S_{1}, \ldots S_{s}$ can be only of the form

$$
S_{\alpha_{1} \beta} \cap S_{\alpha_{2} \beta} \cap S_{\alpha_{3} \beta}=S_{\alpha \beta} \quad\left(\text { or } S_{\beta \alpha_{1}} \cap S_{\beta \alpha 2} \cap S_{\beta \alpha_{3}}=S_{\beta \alpha}\right)
$$

for some $\alpha_{1}^{n}, \alpha_{2}^{n}, \alpha_{3}^{n}, \beta^{n}, \alpha \in T, \alpha=\alpha_{1} \cap \alpha_{2} \cap \alpha_{3}$. Since $S_{\alpha \beta} \neq \emptyset$, it follows that $\operatorname{dim} \alpha=n-1$. Since $N$ is a closed manifold, then no three distinct $n$-simplices of $T$ intersect by an $(n-1)$-simplex of $T$. This contradiction shows that a) is true.

Define $A_{i}$ as in 2.2. If $S_{i} \cap S_{j} \neq \emptyset$, then take a point $a_{i j} \in S_{i} \cap S_{j}$ and let $A_{i j}=\mathrm{st}^{2} a_{i j}$. From definition of $S_{i}$ and $A_{i}$ it follows that $S \subset A_{i}$ and $A_{i} \cup A_{j} \subset A_{i j}$. By the choise of $T, A_{i}$ and $A_{i j}$ are contractible.

2.4. Decomposition of $S(f)$ for $d=2$. Let $N$ be a closed $P L n$-manifold and $m=\frac{3 n}{2} \geq n+3$. Then there is a triangulation $T$ of $N$ such that for every map $f: N \rightarrow \mathbb{R}^{m}$, satisfying to (2.1.1.a,b) there are subpolyhedra $S_{1}, \ldots, S_{s} \subset N$ such that $S(f)=S_{1} \cup \cdots \cup S_{s}$ and

a) The intersections $S_{i} \cap S_{j} \cap S_{k}$ are either disjoint or the same for distinct sets $\{i, j, k\} \subset\{1, \ldots, s\}$.

b) For each $i=1, \ldots, s$ there is a contractible polyhedron $A_{i} \subset N$ containing $S_{i}$. If $S_{i} \cap S_{j} \neq \emptyset$ then there is a contractible polyhedron $A_{i j} \subset N$ containing $A_{i} \cup A_{j}$. If $S_{i} \cap S_{j} \cap S_{k} \neq \emptyset$, then there is a contractible polyhedron $A_{i j k} \subset N$, containing $A_{i j} \cup A_{j k} \cup A_{k i}$.

Proof. Take a triangulation $T$ of $N$ such that for each $x \in N$, the $\operatorname{star} \mathrm{st}^{4} x$ is contractible. Let $S_{1}, \ldots, S_{s}$ be as defined in 2.2. We shall prove an assertion, equivalent to (2.4.a): if $S_{i_{1}} \cap \cdots \cap S_{i_{k}} \neq \emptyset$ for some $k \geq 4$, then every three of $S_{i_{1}} \ldots S_{i_{k}}$ have the same intersection.

By 2.1.1.b $S_{\alpha \beta} \neq \emptyset$ is possible only when $\{\operatorname{dim} \alpha, \operatorname{dim} \beta\}$ is either $\{n, n\}$ or $\{n, n-1\}$ or $\{n, n-2\}$. By general position, $f$ has no quadruple points and each of its triple points is intersection of $f$-images of three $n$-dimensional open simplices of $T$. Therefore each intersection of $k \geq 4$ of $S_{1}, \ldots, S_{s}$ is of the form

$$
S_{\alpha_{1} \beta} \cap \cdots \cap S_{\alpha_{k} \beta}=S_{\alpha \beta} \quad\left(\text { or } S_{\beta \alpha_{1}} \cap \cdots \cap S_{\beta \alpha_{k}}=S_{\beta \alpha}\right)
$$

for some $\alpha_{1}^{n}, \ldots, \alpha_{k}^{n}, \beta^{n} \alpha \in T, \alpha=\alpha_{1} \cap \cdots \cap \alpha_{k}$. Consider only the first case, the second is proved analogously. Since $S_{\alpha \beta} \neq \emptyset$ then $\operatorname{dim} \alpha \geq n-2$. If $\operatorname{dim} \alpha=n-1$, then $k=2$, which is a contradiction. Therefore $\operatorname{dim} \alpha=n-2$. The intersection of 
every three distinct simplices $\alpha_{p}, \alpha_{q} \alpha_{r}$ from $\left\{\alpha_{1}, \ldots, \alpha_{k}\right\}$ contains $\alpha$ and cannot be $(n-1)$-dimensional, hence $\alpha_{p} \cap \alpha_{q} \cap \alpha_{r}=\alpha$. Since $f$ has no quadruple points, it follows that $S_{\alpha_{p} \beta} \cap S_{\alpha_{q} \beta} \cap S_{\alpha_{r} \beta}=S_{\alpha \beta}$ and we are done.

The polyhedra $A_{i}$ and $A_{i j}$ are defined as in 2.3, 2.4. If $S_{i} \cap S_{j} \cap S_{k} \neq \emptyset$, then take a point $a_{i j k} \in S_{i} \cap S_{j} \cap S_{k}$ and let $A_{i j k}=$ st $^{4} a_{i j k}$. By the choise of $T$, we have that $A_{i}, A_{i j}$ and $A_{i j k}$ are contractible. Then (2.4.b) is proved similarily to (2.3.b).

2.5. Homotoping $S(f)$ onto its 'reduced' nerve. Let $d \in\{1,2\}, N$ be a polyhedron and $S_{1}, \ldots, S_{s}$ its subpolyhedra, satisfying $(2 . d+2 . a)$ and $(2 . d+2 . b)$. Then the inclusion $\bigcup_{i=1}^{s} S_{i} \subset N$ is homotopic to a map in some d-dimensional subpolyhedron of $N$.

Proof. Proof for the case $d=2$ is analogous to that for $d=1$. Suppose that $d=1$. From (2.3.1) it follows that the sets $S_{i} \cap S_{j}$ are disjoint for distinct nonordered pairs $i, j=1, \ldots, s$. Take disjoint regular neighborhoods $U_{i j}$ of $S_{i} \cap S_{j}$ in $\bigcup_{i=1}^{s} S_{i}$. Since $A_{i}$ is contractible, it follows that there is a homotopy $F_{i}$ :

$\mathrm{Cl}\left(S_{i}-\bigcup_{j \neq i} U_{i j}\right) \times I \rightarrow A_{i}$ between the inclusion and a constant map to some point $a_{i} \in A_{i}$.

Suppose that $S_{i} \cap S_{j} \neq \emptyset$. Since $A_{i j}$ is contractible, it follows that there is an $\operatorname{arc} l_{i j} \subset A_{i j}$, joining $a_{i}$ and $a_{j}$. Also we can extend homotopies $F_{i}$ and $F_{j}$ over $U_{i j}$ to a homotopy $F_{i j}: U_{i j} \times I \rightarrow N$ between the inclusion and a map of $U_{i j}$ to $l_{i j}$ (we can do this by simplices beginning with the 0-dimensional ones). Since all $U_{i j}$ are disjoint and all $F_{i j}$ are extensions of $F_{i}$ and $F_{j}$, it follows that all constructed homotopies define a homotopy $F:\left(\bigcup_{i=1}^{s} S_{i}\right) \times I \rightarrow N$ between the inclusion and a map onto the subgraph $\left(\bigcup_{i=j}^{s} a_{i}\right) \cup\left(\bigcup\left\{l_{i j} \mid 1 \leq i<j \leq s\right.\right.$ and $\left.\left.S_{i} \cap S_{j} \neq \emptyset\right\}\right)$ of $N$.

\section{Proof of theorems 1.1.bc}

Theorem 1.1.b is proved analogously to theorem 1.1.a, using the following theorems 3.1 and 3.2 instead of theorems 2.1.1 and 2.1.3, respectively. Theorem 3.1 is proved analogously to theorem 2.1 .1 by induction on pairs $(\sigma, \tau)$ of simplices of $T-A$. Theorem 3.2 is proved analogously to theorem 2.1.3.

Theorem 3.1. Suppose that $N$ is an n-polyhedron with triangulation $T, A$ is a subpolyhedron of $N, g: N \rightarrow B^{m}$ is a PL map such that $\left.g\right|_{A}$ is a PL-embedding into $\partial B^{m}$ and $g(N-A) \subset \stackrel{\circ}{B^{m}}$. If $m-n \geq 3$ and the equivariant map $\tilde{g}: A^{*} \rightarrow S^{m-1}$ extends to an equivariant map $F: \tilde{N} \rightarrow S^{m-1}$, then there exists a general position $P L$-extension $f: N \rightarrow \mathbb{R}^{m}$ of $\left.g\right|_{A}$ such that

a) $\left.f\right|_{\sigma}$ is embedding for each $\sigma \in T$;

b) $f \sigma \cap f \tau=f(\sigma \cap \tau)$ whenever $\operatorname{dim} \tau \leq \operatorname{dim} \sigma$ and $2 \operatorname{dim} \sigma+\operatorname{dim} \tau \leq 2 m-3$;

c) $f(N-A) \subset \stackrel{\circ}{B}^{m}$. 
Theorem 3.2. Suppose that $N$ is a d-connected $P L$ n-manifold with boundary, $m-n \geq 3$ and there is a PL map $g: N \rightarrow B^{m}$ such that $\left.g\right|_{\partial N}$ is an embedding into $\partial B^{m}, g \stackrel{\circ}{N} \subset \stackrel{\circ}{B}^{m}$ and $S(g)=C l\left\{x \in N:\left|g^{-1} g x\right|>1\right\}$ is contained in some $P L n$-ball in $\stackrel{\circ}{N}$. Then there is an embedding $f: N \rightarrow B^{m}$ such that $\left.f\right|_{\partial N}=\left.g\right|_{\partial N}$.

Theorem 1.1.c is deduced from theorem 1.1.b analogously to [We 67, §7], only one should use boundary version 1.1.b (but not relative version). To cover the inaccuracy of [We 67, §7], mentioned in the introduction, and for completeness, we present the proof here.

Proof of theorems 1.0.e and 1.1.c. Let $g: N \times I \rightarrow I^{m} \times I$ be the linear homotopy between $f_{0}$ and $f_{1}$. By lemma 3.3 below there exists an equivariant map $H$ : $\widetilde{N \times I \rightarrow S^{m}}$ such that $H_{(N \times 0 \cup N \times 1)^{*}}$ is equivariantly homotopic to $\tilde{g}_{(N \times 0 \cup N \times 1)^{*}}$. Applying theorem 1.0.c or 1.1.b for the PL-manifold $N \times I$, its subpolyhedron $N \times 0 \cup N \times 1$ and the map $g$, we obtain an embedding $\varphi: N \times I \rightarrow I^{m} \times I$ such that $\varphi(x, 0)=f_{0}(x)$ and $\varphi(x, 1)=f_{1}(x)$. By definition, $\varphi$ is a concordance between $f_{0}$ and $f_{1}$. By the 'concordance implies isotopy' theorem [Li 65] (see also [Hu 70]), $f_{0}$ and $f_{1}$ are isotopic.

Lemma 3.3. (cf. [We 67, Lemma 7]) Suppose that $N$ is a space, $g: N \times I \rightarrow$ $I^{m} \times I=I^{m+1} \subset \mathbb{R}^{m}$ is a map such that $g(N \times(0,1)) \subset I^{m} \times(0,1),\left.g\right|_{N \times 0}$ and $\left.g\right|_{N \times 1}$ are embeddings into $I^{m} \times 0$ and $I^{m} \times 1$, respectively, and there exists an equivariant homotopy $h: \tilde{N} \times I \rightarrow S^{m-1}$ between $\left.g\right|_{N \times 0}$ and $\left.g\right|_{N \times 1}$. Then $\tilde{g}$ is defined over $(N \times 0 \cup N \times 1)^{*}$ and there exists an equivariant map $H: N \times I \rightarrow S^{m}$ such that $\left.H\right|_{(N \times 0 \cup N \times 1)^{*}}$ is equivariantly homotopic to $\left.\tilde{g}\right|_{(N \times 0 \cup N \times 1)^{*}}$.

Idea of the proof. The construction of $H$ is illustrated by Figure 1. The deleted product $N \times I$ is shown as the cube $A B C D A^{\prime} B^{\prime} C^{\prime} D^{\prime}$ without the $\operatorname{arc} P Q . \tilde{N} \times$ $(0,0)$ and $\tilde{N} \times(1,1)$ are shown as the $\operatorname{arc} A A^{\prime}$ without the point $P$ and the $\operatorname{arc} C C^{\prime}$ without the point $Q$, respectively. The image of $\tilde{N} \times I$ under the standard inclusion into $\widetilde{N \times I}$ is $\tilde{N} \times \operatorname{diag} I$ and is shown as the section $A C C^{\prime} A^{\prime}$ without the $\operatorname{arc} P Q$. The part $(N \times 0 \cup N \times 1)^{*}$, on which $H$ and $\tilde{f}$ should be homotopic, is shown as the union of the four faces

$$
A D D^{\prime} A^{\prime} \cup D C C^{\prime} D^{\prime} \cup C B B^{\prime} C^{\prime} \cup B A A^{\prime} B^{\prime} .
$$

The pyramid $P Q D D^{\prime}$ without the arc $P Q$ goes to the north pole $R$. The symmetric pyramid $P Q B B^{\prime}$ without the $\operatorname{arc} P Q$ goes to the south pole $S$. On the section $A C C^{\prime} A^{\prime}$ without the $\operatorname{arc} P Q, H$ equals to $h$. Then $H$ is extended 'linearly' to the upper (lower) half-cube $A A^{\prime} B B^{\prime} C C^{\prime}\left(A A^{\prime} B B^{\prime} C C^{\prime}\right)$ and send it to the northern (southern) hemisphere.

Accurate proof. We follow [We 67, proof of lemma 7]. Denote by $R$ and $S$ the 
north and the south pole of the sphere $S^{m}$, respectively. For a vector $r \in \mathbb{R}^{m+1}$

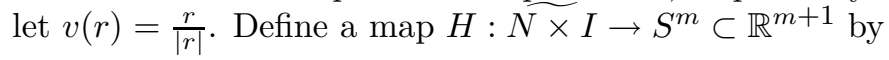

$$
H(x, s, y, t)= \begin{cases}S, & s-t \geq|x, y| \\ v\left(\frac{s-t}{|x, y|} S+\left(1-\frac{s-t}{|x, y|}\right) h\left(x, y, \frac{s+t}{2}\right)\right), & 0 \leq s-t<|x, y| \\ v\left(\frac{t-s}{|x, y|} R+\left(1-\frac{t-s}{|x, y|}\right) h\left(x, y, \frac{s+t}{2}\right)\right), & 0 \leq t-s<|x, y| \\ R, & t-s \geq|x, y|\end{cases}
$$

It is easy to see that $H$ is equivariant. Also, $H(x, t, y, t)=h(x, y, t)$, hence $H_{\widetilde{N \times 0} \cup \widetilde{N \times 1}}$ is equivariantly homotopic to $\tilde{g}_{\widetilde{N \times 0} \cup \widetilde{N \times 1}}$. For $(x, t, y, 1) \in N \times[0,1) \times$ $N \times\{1\}$, both $H(x, t, y, 1)$ and $\tilde{g}(x, t, y, 1)$ are in the northern open hemisphere. For $(x, t, y, 0) \in N \times[0,1) \times N \times\{0\}$, both $H(x, t, y, 0)$ and $\tilde{g}(x, t, y, 0)$ are in the southern open hemisphere. Therefore for each

$$
(x, s, y, t) \in(N \times 0 \cup N \times 1)^{*}-(\widetilde{N \times 0} \cup \widetilde{N \times 1}),
$$

points $H(x, s, y, t)$ and $\tilde{g}(x, s, y, t)$ are not antipodal. Therefore $H_{(N \times 0 \cup N \times 1) *}$ is equivariantly homotopic to $\tilde{g}_{(N \times 0 \cup N \times 1)^{*}}$.

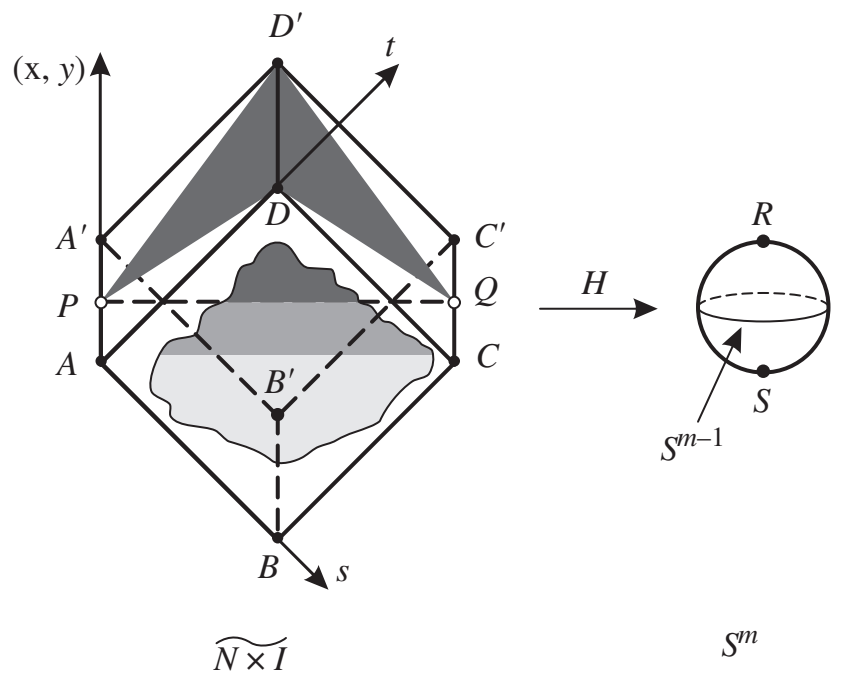

Figure 1. 


\section{Conjectures}

It would be interesting to prove smooth analogue of theorem 1.1.a. It would also be interesting to generalize theorem 1.1 to embeddings of a manifold with boundary into a manifold with boundary, cf. [Har 69]. Theorem 1.0.b suggests

Conjecture 4.1. (cf. [Sk]) If $N$ is an n-manifold and $m=\frac{3 n}{2}+1$ (in particular, $(m, n)=(4,2))$, then for each equivariant map $F: \tilde{N} \rightarrow S^{m-1}$ there exists an embedding $f: N \rightarrow \mathbb{R}^{m}$ such that $\tilde{f}$ is equivariantly homotopic to $F$.

Problem 4.2. (cf. corollary 1.4.b) Is every orientable 6-manifold embeddable in $\mathbb{R}^{10}$ ?

Idea of proof of theorem 1.1 and [Hu 67] motivate

Conjecture 4.3. (cf. [Sk]) a) Suppose that $m \geq n+3$ and $N$ is a d-connected closed $P L n$-manifold. Then $N$ is embeddable in $\mathbb{R}^{m}$ if and only if there exist an equivariant map $F: \tilde{N} \rightarrow S^{m-1}$;

b) the above is true even without the connectedness assumption.

The idea of $\S 2$ works to prove conjecture 4.3.a for $d=3$. Using finger moves as in [ST 91], one can prove conjecture 4.3.a for $d=4,5$. But for $d>5$ this approach requires stronger connectivity assumption. Note that conjecture 4.3.a is true for PL-embeddings when $m<n+3$ since for this case $N$ is either $n$-connected (which is not possible) or a homotopy sphere. Then conjecture 4.3.a is true for $n=3$ by [Wa 65 ] and for $n \geq 4$ by [Ker 69], cf case $n=4$ in the beginning of 2.1. For PL-embeddings and if $m<\frac{5 n+6}{4}$, we have $d>\frac{n}{2}-1$, hence $N$ is a homotopy sphere, and conjecture 4.3.a, however true, is not interesting. In [Gor 72] it was pointed out that the condition of $(3 n-2 m+2)$-connectedness is unnecessary in [Hu 67]. This motivies conjecture 4.3.b.

\section{Acknowledgements}

I would like to acknowledge P. M. Akhmetyev, A. Szucs and A. V. Zhubr for useful discussions. These results were first presented at The Alexandrov Sessions in Moscow (May, 1996 and May, 1997) and at The Borsuk-Kuratowski Session in Warsaw (May 1996).

\section{References}

[BH 70] J. Boechat, A. Haeflinger, Plongements differentiables de varietes de dimension 4 dans $\mathbb{R}^{7}$. Essays on topology and related topics Springer, 1970. 
[BH 71] J. Boechat, A. Haeflinger, Plongements differentiables de varietes de dimension $4 k$ dans $\mathbb{R}^{6 k+1}$, Comment. Math. Helv. 46(2) (1971), 141-161.

[Br 72] J. L. Bryant, Approximating embeddings of polyhedra in codimension 3, Trans. AMS 170 (1972), 85-95.

[Ch 69] A. V. Chernavskii, Piecewise linear approximations of embeddings of cells and spheres in codimensions higher than two, Mat. Sb. 80 (1969), no. 122, 339-364; Math. USSR Sb. 9 (1969), 321-344.

[CS 79] S. E. Cappell, J. L. Shaneson, Imbeddings and immersions of 4-dimensional manifolds in $\mathbb{R}^{6}$, Geometric Topology, J. C. Cantrell (eds.), Academic Press, New York 1979, pp. 301-303.

[FF 89] A. T. Fomenko, D. B. Fuchs, A course in homotopy topology, Nauka, Moscow 1989 (in Russian).

[FKT 94] M. H. Freedman, V. S. Krushkal, P. Teichner, Van Kampen's embedding obstruction is incomplete for 2-complexes in $\mathbb{R}^{4}$, Math Res. Letters 1 (1994), 167-176.

[FU 94] F. Fuquan, Embedding four manifolds in $\mathbb{R}^{7}$, Topology 33(3) (1994), 447-454.

[Gor 72] C. M. A. Gordon, Embeddings of PL-manifolds with boundary, Procl Camb. Phil. Soc. 72 (1972), 21-25.

[Hae 62] A. Haefliger, Plongements de varietes dans le domain stable, Seminare Bourbaki 245 (1962).

[Hae 63] A. Haefliger, Plongements de varietes dans le domain stable, Comment. Math. Helv. 36 (1962-63), 155-176.

[Ha 62] A. Haefliger, Knotted (4k-1)-spheres in 6k-space, Ann. of Math. 75 (1962), $452-466$.

[Har 69] L. S. Harris, Intersections and embeddings of polyhedra, Topology 8 (1969), 1-26.

[Hi 61] M. W. Hirsch, The embedding of bounding manifolds in euclidean spaces, Ann. of Math. 74 (1961), 494-497.

[Hi 65] M. W. Hirsch, On embedding 4-manifold in $\mathbb{R}^{7}$, Proc. Camb. Phil. Soc. 61(3) (1965), 657-658.

[Hu 63] J. F. P. Hudson, Knotted tori, Topology 2 (1963), 11-23.

[Hu 67] J. F. P. Hudson, PL embeddings, Ann. of Math. (2) 85(1) (1967), 1-31.

[Hu 69] J. F. P. Hudson, Piecewise-Linear Topology, Benjamin, New York-Amsterdam 1969.

[Hu 70] J. F. P. Hudson, Concordance, isotopy and diffeotopy, Ann. of Math. (3) 91(3) (1970), 425-448.

[Hu 72] J. F. P. Hudson, Embeddings of bounded manifolds, Proc. Camb. Phil. Soc. 72 (1972), 11-20.

[Hus 88] L. S. Husch, $\epsilon$-maps and embeddings, General Topological Relations to Modern Analysis and Algebra VI, Heldermann, Berlin 1988, pp. 273-280.

[Ir 65] M. C. Irwin, Embeddings of polyhedral manifolds, Ann. of Math. (2) 82 (1965), $1-14$.

[Ke 69] M. A. Kervaire, Smooth homotopy spheres and their fundamental groups, Trans. Amer. Math. Soc. 144 67-72.

[Li 65] W. B. R. Lickorish, The piecewise linear unknotting of cones, Topology 4 (1965), $67-91$.

[MS 67] S. Mardesic, J. Segal, $\epsilon$-mappings and generalized manifolds, Michigan Math. J. 14 (1967), 171-182.

[Ma 60] W. S. Massey, On the Stiefel-Whitney classes of a manifold, 1, Amer J. Math. 82 (1960), 92-102.

[Ma 62] W. S. Massey, On the Stiefel-Whitney classes of a manifold, 2, Proc. AMS 13 (1962), 938-942.

[Ma 90] W. S. Massey, Homotopy classification of 3-component links of codimension greater than 2, Topol. Appl. 34 (1990), 269-300. 
Vol. 72 (1997) On the deleted product criterion for embeddability of manifolds in $\mathbb{R}^{m}$

[Mi 72] R. T. Miller, Approximating codimensions 3 embeddings, Ann. Math. 95 (1972), 406-416.

[Mi 65] J. Minkus, On embeddings of highly connected manifolds, Trans AMS 115 (1965), 525-540.

[PWZ 61] R. Penrose, J. H. C. Whitehead, E. C. Zeeman, Imbedding of manifold in euclidean space, Ann. of Math. 73 (1961), 613-623.

[Ro 65] V. A. Rohlin, Embedding of non-orientable three-dimensional manifolds in the fine-dimensional euclidean space (in Russian), Dokl. Akad. Nauk SSSR 160 (1965), 549-551.

[RS 72] C. P. Rourke, B. J. Sanderson, Introduction to Piecewise-Linear Topology, Ergebn. der Math. 69 Springer Verlag, Berlin 1972.

[RS 96] D. Repovs and A. B. Skopenkov, Embeddability and isotopy of polyhedra in Euclidean spaces, Proc. of the Steklov Math. Inst. 212 (1996), 163-178.

[RS 97] D. Repovs and A. B. Skopenkov, A deleted product criterion for approximability of maps by embeddings, Topology Appl., to appear.

[Ru 82] D. Ruberman, Imbeddings four-manifolds and slicing links. Math. Proc. Camb. Phil. Soc. 91 (1982), 107-110.

[SS 92] J. Segal and S. Spiez, Quasi-embeddings and embedding of polyhedra in $\mathbb{R}^{m}$, Topol. Appl. 45 (1992), 275-282.

[SSS 97] J. Segal, A. Skopenkov and S. Spiez, Embedding of polyhedra in $\mathbb{R}^{m}$ and the deleted product obstruction, Topol. Appl. 85 (1997), 1-10.

[Sk 97] A. B. Skopenkov, On the deleted product criterion for embeddability in $\mathbb{R}^{m}$, Proc. AMS (1997), to appear.

[Sk 96] A. B. Skopenkov, On the deleted product criterion for embeddability of manifolds in $\mathbb{R}^{m}$, Abstracts AMS (1996), 96T-57-179.

[Sk] A. B. Skopenkov, On the deleted product criterion for embeddability of manifolds in $\mathbb{R}^{m}$, II, preprint.

[Sm 62] S. Smale, On the structure of 5-manifold, Ann. Math. 75 (1962), 38-46.

[ST 91] S. Spiez and H. Toruńzyk, Moving compacta in $\mathbb{R}^{m}$ apart, Topol. Appl. 41 (1991), 193-204.

[Va 92] V. A. Vassiliev, Complements of discriminants of smooth maps, AMS, Providence, RI 1992.

[Wa 65] C. T. C. Wall, All 3-manifolds imbed in 5-space, Bull. AMS 71(3) (1965), 564567.

[We 67] C. Weber, Plongements de polyedres dans le domaine metastable, Comment. Math. Helv. 42 (1967), 1-27.

[Wu 65] W. T. Wu, A Theory of Embeddings, Isotopu and Immersions of Polyhedra in Euclidean Space, Science Press, Peking 1965.

[Ze 62] E. C. Zeeman, The Poincare conjecture for $n \geq 5$, in: Topology of 3-manifolds, ed. M. K. Fort, Prentice Hall 1962.

[Zh 75] A. V. Zhubr, A classification of simply-connected spin 6-manifolds (in Russian), Izvestiya AN SSSR 39(4) (1975), 839-856.

[Zh. 80] A. V. Zhubr, A classification of simply-connected 6-manifolds (in Russian), Doklady AN SSSR 255(6) (1980), 1312-1315.

[Zh. 89] A. V. Zhubr, Classification of simply-connected topological 6-manifolds, Lecture Notes in Math. 1346 (1989), 325-339.

\section{A. Skopenkov}

Chair of Differential Geometry

Department of Mechanics and Mathematics

Moscow State University

Moscow, 119899 Russia

e-mail: skopenko@nw.math.msu.su

(Received: June 27, 1996) 